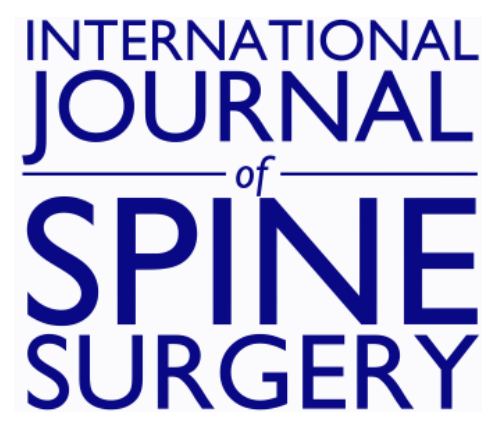

\title{
Can Morphometric Analysis of Cervical Spondylotic Myelopathy Be a Tool for Surgical Outcome Prediction?
}

Sofia Tavares, Gonçalo Guerreiro Costa, Orlando Galego and Ricardo Pereira

Int J Spine Surg 2021, 15 (4) 718-723

doi: https://doi.org/10.14444/8094

http://ijssurgery.com/content/15/4/718

This information is current as of April 25, 2023.

Email Alerts Receive free email-alerts when new articles cite this article. Sign up at:

http://ijssurgery.com/alerts

The International Journal of Spine Surgery

2397 Waterbury Circle, Suite 1,

Aurora, IL 60504, Phone: +1-630-375-1432 


\title{
Can Morphometric Analysis of Cervical Spondylotic Myelopathy Be a Tool for Surgical Outcome Prediction?
}

\author{
SOFIA TAVARES, MMED,${ }^{1}$ GONÇALO GUERREIRO COSTA, MD,${ }^{1}$ ORLANDO GALEGO, MMED, ${ }^{2}$ \\ RICARDO PEREIRA, MMED ${ }^{1}$ \\ ${ }^{I}$ Centro Hospitalar e Universitário de Coimbra, Neurosurgery Department, Coimbra, Portugal, ${ }^{2}$ Centro Hospitalar e Universitário de Coimbra, Neuroradiology \\ Department, Coimbra, Portugal
}

\begin{abstract}
Background: Cervical spondylotic myelopathy (CSM) is one of the leading causes of degenerative cervical myelopathy and the most common cause of spinal cord dysfunction in the elderly worldwide. Although there is emerging evidence that most patients improve after surgery, the key clinical and imaging factors predicting outcome remain uncertain. Our purpose is to evaluate preoperative and postoperative morphometric parameters on magnetic resonance imaging and their relation with neurological outcome at discharge and at 12-month follow-up.

Methods: Morphometric features (volume, area, and antero-posterior diameter of the major stenotic section) were acquired by manual segmentation of the spinal canal using OsiriX open-source software and confronted with neurological outcome (at discharge and 12 months after surgery) using Nurick's scale.

Results: Fifty-five patients (21 females and 34 males) with a mean age of $64.89 \pm 11.95$ years were analyzed. Recovery ratio was $2.44 \% \pm 2.40 \%$ at discharge and $11.74 \% \pm 2.50 \%$ at follow-up. Statistical analysis revealed a significant difference $(P<.001)$ between Nurick's scale at admission versus discharge, at discharge versus follow-up at 1 year, and between admission and follow-up at 1 year. Morphometric changes (difference and ratio) between preoperative and postoperative measurements were also statistically significant $(P<.001$, paired samples $t$ test). When linear regression was applied, volume difference was shown to have an influence on clinical improvement $(P<.05 ; R=$ 0.519). Linear regression was also applied using recovery ratio at discharge and follow-up as dependent variables, with the same conclusion: volume difference between preoperative and postoperative measurements correlates with outcome improvement at 1 year after surgery.

Conclusions: Acquisition of morphometric features might be useful in predicting surgical outcome in patients with CSM. This information can be used to inform patients of their long-term postoperative prognosis in the future with more accurate and standardized measurements.

Cervical Spine

Keywords: degenerative spondylotic myelopathy, magnetic resonance imaging, spinal cord, surgical outcomes
\end{abstract}

\section{INTRODUCTION}

Degenerative cervical spondylotic myelopathy (CSM) is widely recognized as the most common etiology of spinal cord dysfunction and the most frequent cause of spinal cord injury in patients aged $>55$ years. ${ }^{1}$ It usually manifests as a progressive neurological deterioration with cumulative impairments, such as reduction of proprioception, motor weakness, and gait imbalance. ${ }^{1,2}$

The development of CSM is associated with agerelated degeneration of the cervical spine anatomy. ${ }^{3}$ Compressive forces exerted over time on the spinal cord and aberrant migration of tissue into the spinal canal contribute to the development of myelopathy through direct compression, ischemia, and other pathologic processes affecting the spinal cord. ${ }^{4}$
Although patients with CSM with mild symptoms are often managed conservatively, early surgical decompression is increasingly recommended as the standard of care for patients presenting with moderate to severe symptoms. ${ }^{2,3}$ Decisions regarding care of patients with mild to moderate symptoms are far more complex and challenging, being largely dependent on radiological characteristics, clinical presentation, practitioner experience, and patient preference. ${ }^{2}$ There is some evidence that duration of symptoms and possibly advancing age negatively affect outcome, but at present, it is difficult to predict an individual patient's response to surgical intervention. ${ }^{5}$

Surgery of any kind, particularly involving the cervical spine, does not always result in perfect 
outcomes and is associated with risks. ${ }^{3}$ This outcome variability underscores the need to refine surgical decision making. ${ }^{2}$

Advances in magnetic resonance imaging (MRI) acquisition and postprocessing software have enabled the quantification of morphological changes in regions of the spinal cord, and some of these findings have been suggested to be predictive of functional recovery. ${ }^{2,5}$ However, the literature varies on the precise importance of MRI features in relation to prognosis. ${ }^{5}$

MRI-based factors, including T1 hypointensity, T2 hyperintensity, and morphological parameters, may have a role in predicting outcome, although robust evidence is lacking. ${ }^{2}$ However, postoperative MRI has thus far been less extensively studied in relation to the longer-term prognosis in CSM patients. In particular, there are few studies looking at recovery in relation to the morphology of cervical cord compression and its re-expansion. ${ }^{5}$

Most morphological measurements of cervical canal stenosis studies focused only on the radial line length of the cervical spinal canal and the cervical spinal body or the cross-sectional area of the cervical spinal canal, and thus volumetric compression on the cervical spinal cord could not be objectively and accurately examined. ${ }^{6}$

In this retrospective study, we examined preoperative and postoperative MRI and functional assessment using Nurick's scale - an objective assessment of severity of myelopathy more weighted on lower limb function. Score ranges from 0 (least disability) to 5 (most disability).

Our purpose is to evaluate MRI preoperative and postoperative morphometric parameters (volume, area, and antero-posterior diameter) and their correlation with neurological outcome at discharge and at 12-month follow-up and to try to demonstrate their prognostic value for functional recovery following decompression surgery.

\section{METHODS}

A retrospective study of 55 patients with available preoperative and postoperative MRI who were submitted to surgical decompression to treat CSM was developed. Only patients with clear clinical signs of CSM - upper extremity weakness, sensory loss, lack of coordination, and gait instability in combination with documented radiological cervical spinal cord compression-were included. Patients with underlying causes (neurodegenerative diseases

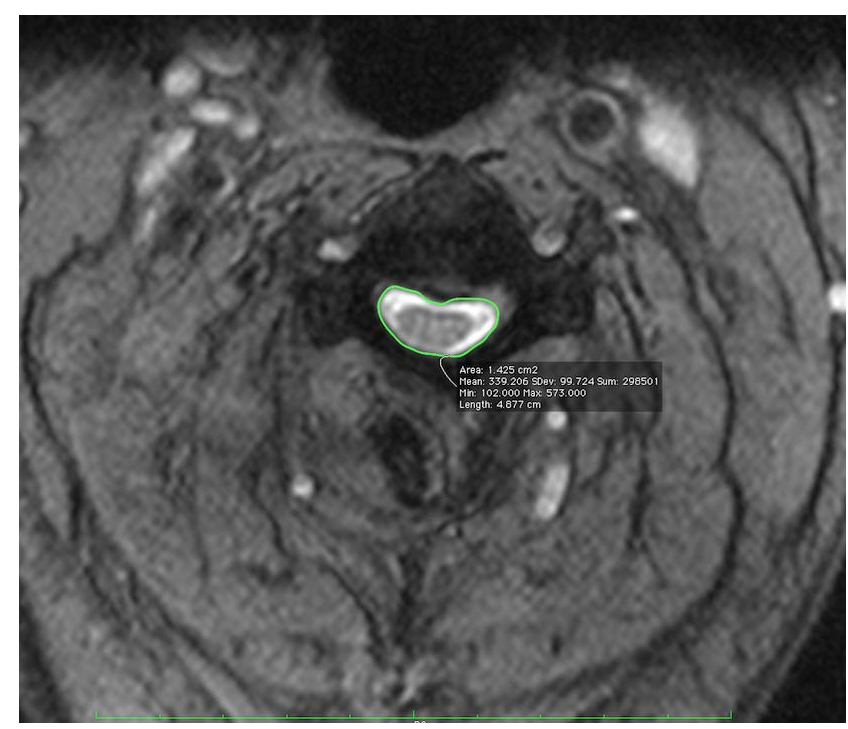

Figure 1. Example of the performed manual segmentation of an axial slice of the spinal cord using OsiriX open-source software.

[Alzheimer's disease or multiple sclerosis] or trauma, tumor, or infection) other than degenerative diseases and patients with a previous history of spinal surgery were excluded.

Clinical state was assessed with Nurick's scale at admission, discharge, and 1 year postoperative. Postoperative MRI study was obtained 3-6 months after surgery.

Spinal canal was segmented using OsiriX opensource software. All axial slices from the stenotic segment were manually segmented (Figure 1), and total volume was automatically computed by the software, taking into account slice thickness and spacing (Figure 2). The same rostral-caudal canal extension was used in preoperative and postoperative images. In the segments corresponding to foramina areas, the lateral limits of segmentation corresponded to the vertical projection on the adjacent medial pedicular wall. These acquisitions were obtained with outcome blinding by an attending neuroradiologist not aware of the patient's clinical status.

\section{Statistical Methods}

All data are described as mean $\pm \mathrm{SD}$, and all statistical data were performed using the Statistical Package for the Social Sciences (SPSS v22.0, SPSS Inc, Chicago, Illinois). Student $t$ tests were performed to assess significant differences between measurements, and chi-square was applied to assess differences in outcome between sex and chosen surgical techniques. Logistic regression and linear 


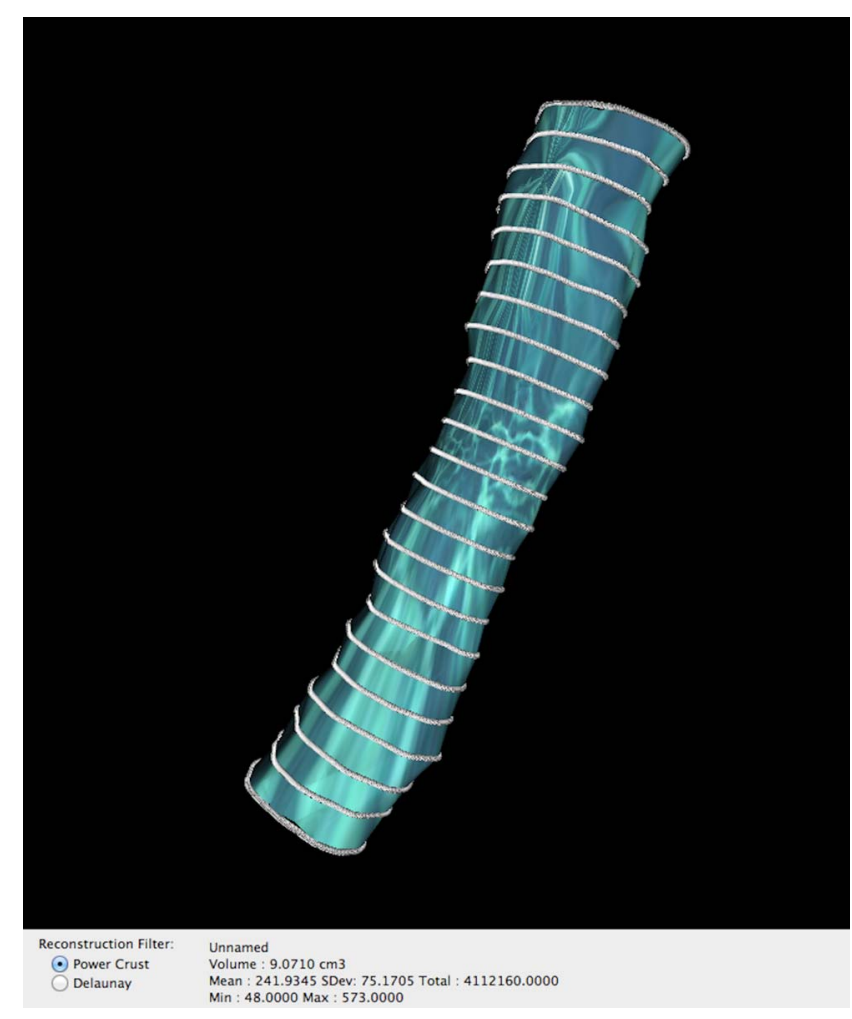

Figure 2. Volume rendering of a major stenotic area after manual segmentation.

regression were applied to assess the influence of morphometric parameters in identifying participants with improvement after surgery. A $P$ value $<.05$ was considered statistically significant.

\section{RESULTS}

\section{Sample Description}

A sample of 55 patients (21 females and 34 males) with a mean age of $64.89 \pm 11.95$ years was analyzed; 36.4\% ( $\mathrm{n}=20)$ were admitted directly from the emergency department for surgery due to a rapid worsening of their neurological deficits. Time of disease progression-between the beginning of symptoms and surgery - was described as months in most patients $(61.8 \%, \mathrm{n}=34)$ as seen in Table 1 .

Nurick preoperative assessment was equal or superior to 2 in $87.3 \%(n=48)$ of patients; $89.1 \%(n$

Table 1. Time of disease progression (since patient awareness of development of symptoms and/or neurological deficits).

\begin{tabular}{lccc}
\hline & Frequency & Percent & Valid Percent \\
\hline Days & 2 & 3.6 & 3.6 \\
Weeks & 9 & 16.4 & 16.4 \\
Months & 34 & 61.8 & 61.8 \\
Years & 10 & 18.2 & 18.2 \\
Total & 55 & 100.0 & 100.0 \\
\hline
\end{tabular}

$=49$ ) had some kind of motor neurological impairment before surgery. The region of major stenosis identified was superior to 2 levels (mean: $2.42 \pm 1.12$ levels $) ; 49.1 \%(n=27)$ of patients were submitted to anterior decompression and $50.9 \%$ (n $=28$ ) to laminectomy with or without fusion. The overall complication rate was of $10.9 \%$. Three patients $(5.45 \%)$ had a slight aggravation of their previous deficits in the early postoperative period with swift recovery. One patient $(1.82 \%)$ was reoperated on 48 hours after for a postoperative epidural hematoma, and 2 patients $(3.64 \%)$ needed a review of the surgical wound for superficial soft tissue infection and wound dehiscence.

In general, $57.1 \%(\mathrm{n}=28)$ of patients revealed clinical improvement (defined as any kind of improvement of motor function or gait) at discharge and $75.0 \%(\mathrm{n}=30)$ at 1-year follow-up. Recovery ratio, defined as Nurick's scale at admission Nurick's scale at discharge or 1-year follow-up/ Nurick's scale at admission, was $12.44 \% \pm 2.40 \%$ at discharge and $11.74 \% \pm 2.50 \%$ at follow-up. Table 2 and Figure 3 summarize the acquired data.

\section{Statistical Analysis}

Statistical analysis revealed a significant $(P<$ .001) difference between Nurick's scale at admission versus discharge, at discharge versus follow-up at 1 year, and between admission and follow-up at 1 year (Wilcoxon signed-rank test).

No difference between sexes in outcome was noted whether at discharge $\left(\chi^{2}, P=.729\right)$ or at 1 year follow-up $\left(\chi^{2}, P=.588\right)$. Also, the chosen surgical technique did not reveal any influence in outcome $\left(\chi^{2}\right.$, outcome at discharge, $P=.824 ; \chi^{2}, 1$ year follow-up, $P=.154)$.

Compared measurements between morphometric features before surgery (antero-posterior diameter, area, and volume) and postoperative measurements were also statistically significant (paired sample $t$ test, $P<.001)$. An increment ratio, defined as postoperative value - preoperative value/postoperative value $\times 100$, was noticed between preoperative and postoperative measures: $21.11 \% \pm 86.40 \%$ for antero-posterior diameter, $39.35 \% \pm 19.98 \%$ for area, and $29.57 \% \pm 17.63 \%$ for volume.

To understand if any relation could be sought between outcome (defined by Nurick's scale) and morphometric measurements (difference and ratio), linear regression was applied using Nurick's scale at 
Table 2. Descriptive statistics of the collected data.

\begin{tabular}{|c|c|c|c|c|c|}
\hline & $\mathbf{N}$ & Minimum & Maximum & Mean & SD \\
\hline Age & 55 & 29 & 82 & 64.89 & 11.948 \\
\hline Number of levels with medullary compression & 55 & 1 & 4 & 2.42 & 1.117 \\
\hline Diameter of maximum compression site & 50 & 2.10 & 7.80 & 4.7358 & 1.54680 \\
\hline Post diameter of maximum compression site & 54 & 1.07 & 13.40 & 7.6098 & 2.34298 \\
\hline Area of maximum compression site & 50 & 17.00 & 148.30 & 70.5520 & 25.12912 \\
\hline Post area of maximum compression site & 54 & 70.00 & 260.00 & 119.6946 & 38.19944 \\
\hline Volume of maximum compression site & 50 & 0.40 & 12.48 & 4.5105 & 2.91674 \\
\hline Post of volume of maximum compression site & 47 & 0.62 & 14.83 & 6.2500 & 3.60587 \\
\hline Diameter difference & 49 & -5.10 & 9.00 & 2.7559 & 2.48894 \\
\hline Diameter ratio & 49 & 0.19 & 5.77 & 0.7889 & 0.86403 \\
\hline Area difference & 49 & 1.60 & 167.30 & 49.3757 & 34.32665 \\
\hline Area ratio & 49 & 0.15 & 0.99 & 0.6065 & 0.19976 \\
\hline Volume difference & 44 & -0.14 & 6.52 & 1.7618 & 1.62804 \\
\hline Volume ratio & 44 & 0.26 & 1.01 & 0.7043 & 0.17632 \\
\hline Recovery ratio, discharge & 54 & -0.33 & 1.00 & 0.1244 & 0.24230 \\
\hline Recovery ratio, 1 y & 47 & -0.33 & 1.00 & 0.1174 & 0.25041 \\
\hline
\end{tabular}

discharge and at 1-year follow-up as dependent variables.

Linear regression was applied to all acquired morphometric differences and ratios with outcome

a

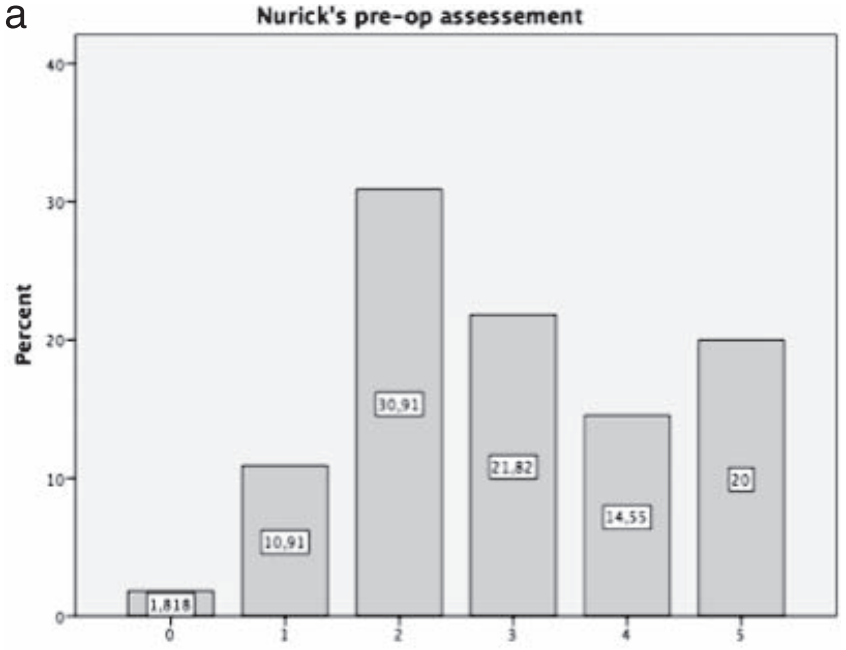

at discharge and at follow-up as dependent variables. We have not found a correlation between measurements and Nurick's scale at discharge, but when Nurick's scale at 1-year follow-up was applied

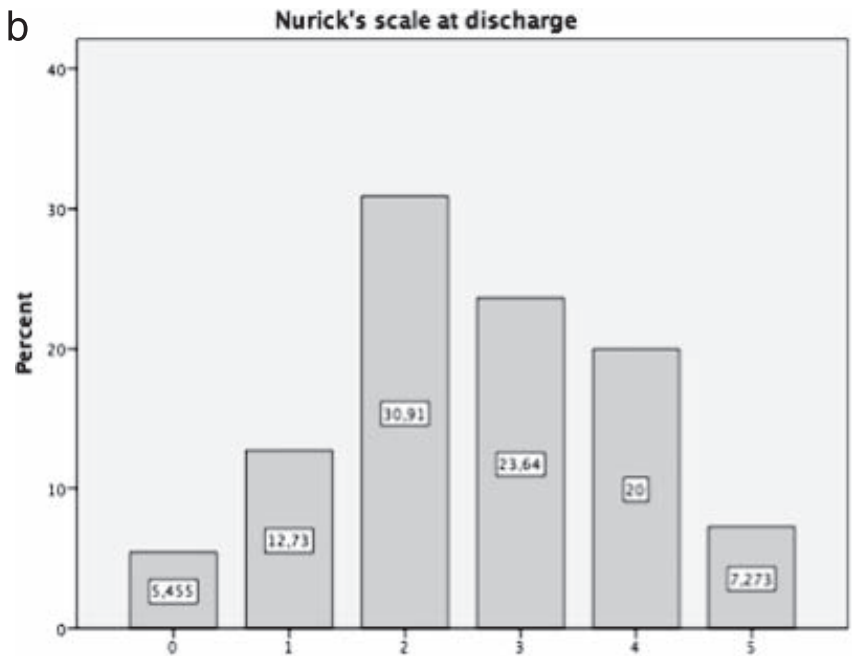

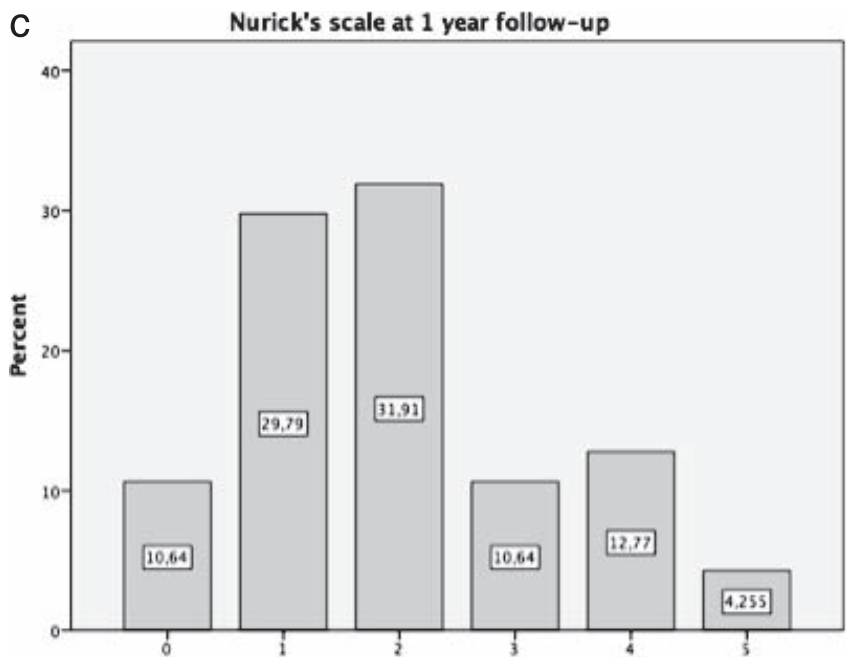

Figure 3. Nurick's scale grades by percentage at (a) admission, (b) discharge, and (c) reevaluation at 1 year postoperative. 
as a dependent variable, volume difference was shown to have an influence on clinical improvement $(P<.05, R=0.519)$ (higher differences between preoperative and postoperative volumes corresponded to a better outcome).

The same statistical examination was applied using the recovery ratio, calculated as Nurick's initial assessment - Nurick's at discharge or followup/Nurick's initial assessment, which at discharge and follow up as dependent variables, with the same conclusion; volume difference between preoperative and postoperative measurements correlated with outcome improvement (the recovery ratio) 1 year after surgery.

\section{DISCUSSION}

In the typical patient with CSM, MRI of the cervical spine provides static images of spinal cord compression resulting from degenerative anatomical changes consequent to the aberrant migration of soft tissue and bone into the spinal canal. ${ }^{3}$ Insult to the cord may be further exacerbated by dynamic phenomena resulting from hypermobility and cervical joint instability. ${ }^{3}$

Numerous investigators have tried to understand the association between various preoperative MRI parameters and postsurgical outcome in patients with CSM; however, their collective results have provided an unclear picture in terms of predictive value and practical application., ${ }^{3,7}$ In addition, there are few studies comparing preoperative and postoperative morphometric features, especially in relation to spinal cord re-expansion.

A systematic review ${ }^{7}$ summarizes several MRI parameters in patients submitted to surgery and in patients with failed conservative treatment, but it failed to identify reproducible features that could strongly relate to outcome. Issues of particular note include variability in approaches used to analyze and quantify structural changes as well as a lack of accepted standard protocols with respect to MRI assessment methodology. ${ }^{3}$

Our goal was to analyze MRI morphometric changes between preoperative and postoperative exams and to try to correlate them with outcome. We verified that surgical decompression was successfully achieved in the majority of cases, with a statistical significance between preoperative and postoperative morphometric parameters. In relation to outcome, we verified that the majority of patients in this series clinically improved after surgery. We did not find any influence of variables such as gender or surgical procedure on outcome.

Volume-occupying rate of cervical spinal canal has already been defined as an objective reflection of compression on the cervical spine and spinal cord and is associated with cervical spinal cord function. ${ }^{6}$ Although differences between preoperative and postoperative diameter and area of major stenotic regions were not found as influencers of outcome in our study, we verified that changes between preoperative and postoperative volume of the stenotic zone were related to neurological outcome improvement at 1-year follow-up. This information reinforces our initial idea that MRI morphometric value alterations before and after surgery can eventually be used to help predict outcome.

However, our study was limited by several factors. This being a retrospective study, we did not have the opportunity to assess more valuable tools to evaluate outcome, such as the modified Japanese Orthopaedic Association scale, which could provide us with more precise information about our patients' neurological states. Nurick's scale presents a clear limitation because it focuses mainly on lower limb function, does not provide a complete evaluation of the patient's neurological status, and has a ceiling effect in patients with initial grade 5 status, making it impossible to describe postoperative improvements in this particular group. Furthermore, MRI images were not always acquired with the same equipment, possibly affecting measurement uniformity.

More information could be brought to light with a controlled prospective study with more individuals, including other tools of clinical evaluation and eventually a combinatorial assessment of other MRI parameters, such as T2 hypersignal measurements, that could yield a greater predictive performance. ${ }^{3}$

The application of newer techniques and technologies, including computational models in the assessment of surgical outcome in patients with CSM, may allow for the improvement of prediction models. ${ }^{3}$ Recent studies have reported the utility of diffusion tensor imaging as an imaging biomarker for the severity of myelopathy and for subsequent surgical outcomes in patients with $\mathrm{CSM}^{4,9}$ by its ability to detect microstructural abnormalities beyond the resolution of conventional MRI and to assess the degree of spinal cord impairment. ${ }^{10}$ Upright and dynamic/kinetic MRI is also a feature to be unveiled that may uncover critical information 
from which new prognostic factors might be derived. $^{3,11}$

\section{CONCLUSIONS}

Acquisition of morphometric features might be useful in predicting surgical outcome in patients with CSM. The fact that volume difference between preoperative and postoperative imaging has a relation with outcome reinforces the idea that this is something to explore in prospective, controlled studies and to combine with newer imaging features in the quest to find a reproducible and reliable way of informing patients of their long-term postoperative prognosis. However, other specific individual and clinical features should never be put aside.

\section{REFERENCES}

1. Sun L-Q, Li YM, Wang X, et al. Quantitative magnetic resonance imaging analysis correlates with surgical outcome of cervical spondylotic myelopathy. Spinal Cord. 2015;53(6):488493. doi:10.1038/sc.2014.204

2. Paliwal M, Weber KA, Hopkins BS, et al. Magnetization transfer ratio and morphometrics of the spinal cord associates with surgical recovery in patients with degenerative cervical myelopathy. World Neurosurg. doi:10.1016/j.wneu.2020.09.148

3. Nouri A, Tetreault L, Zamorano JJ, et al. Role of magnetic resonance imaging in predicting surgical outcome in patients with cervical spondylotic myelopathy. Spine. 2015;40:171-178. doi:10.1097/BRS.0000000000000678

4. Jones JG, Cen SY, Lebel RM, et al. Diffusion tensor imaging correlates with the clinical assessment of disease severity in cervical spondylotic myelopathy and predicts outcome following surgery. Am. J. Neuroradiol. 2013;34:471478. doi:10.3174/ajnr.A3199

5. Arvin B, Kalsi-Ryan S, Karpova A, et al. Postoperative magnetic resonance imaging can predict neurological recovery after surgery for cervical spondylotic myelopathy: a prospective study with blinded assessments. Neurosurgery. 2011;69:362-368. doi:10.1227/NEU.0b013e31821a418c

6. Dong F, Shen C, Jiang S, et al. Measurement of volume- occupying rate of cervical spinal canal and its role in cervical spondylotic myelopathy. Eur Spine J. 2013;22:1152-1157. doi:10.1007/s00586-012-2622-3

7. Tetreault LA, Dettori JR, Wilson JR, et al. Systematic review of magnetic resonance imaging characteristics that affect treatment decision making and predict clinical outcome in patients with cervical spondylotic myelopathy. Spine. 2013;38:110. doi:10.1097/BRS.ObO13e3182a7eaeO

8. Vedantam A, Rajshekhar V. Does the type of T2-weighted hyperintensity influence surgical outcome in patients with cervical spondyloticmyelopathy? A review. Eur Spine J. 2012;22:96-106. doi:10.1007/s00586-012-2483-9

9. Kitamura M, Maki S, Koda M, Satoshi Maki, et al. Longitudinal diffusion tensor imaging of patients with degenerative cervical myelopathy following decompression surgery. $J$ Clin Neurosci. 2020;74:194-198. doi:10.1016/j.jocn.2019.05.018

10. Nakamura M, Fujiyoshi K, Tsuji O, et al. Clinical significance of diffusion tensor tractography as a predictor of functional recovery after laminoplasty in patients with cervical compressive myelopathy. J Neurosurg Spine. 2012;17:147-152. doi:10.3171/2012.5.SPINE1196

11. Jinkins JR, Dworkin JS, Damadian RV. Upright, weight-bearing, dynamic-kinetic MRI of the spine: initial results. Eur Radiol. 2005;15:1815-1825. doi:10.1007/s00330005-2666-4

Disclosures and COI: None of the authors has any conflict of interest to disclose.

Corresponding Author: Sofia Tavares, MMed, Centro Hospitalar e Universitário de Coimbra, Neurosurgery Department, Coimbra, Portugal. Phone: +351917565117; Fax: $(+351)$ 239400400; Email: sofia.icpg.tavares@gmail.com.

Published 19 August 2021

This manuscript is generously published free of charge by ISASS, the International Society for the Advancement of Spine Surgery. Copyright (c) 2021 ISASS. To see more or order reprints or permissions, see http://ijssurgery.com. 